\title{
Massenproduktion ...
}

Für die Massen produzieren Unternehmen seit der industriellen Revolution. Dass sich Firmen der Massen bedienen, um zu produzieren, ist ein relativ neues Phänomen. Das Prinzip, Leistungen durch eine große Zahl von Internetnutzern erbringen zu lassen, kennen die meisten durch Wikipedia - die sogenannte "Wisdom of the Crowds“ sorgt dabei dafür, dass vergleichsweise hohe Qualität entsteht, obwohl die Einträge nicht wie bei klassischen Lexika grundsätzlich von Experten verfasst werden.

Während Wikipedia auf den Idealismus und die Begeisterung Freiwilliger setzt, ist Crowdsourcing im professionellen



Peter Pagel

Chefredakteur Umfeld längst ein Geschäft geworden. Das Kunstwort „Crowdsourcing“ setzt sich zusammen aus „Crowd“, also Menschenmenge, und Outsourcing. Crowdsourcing-Anbieter stellen Plattformen zur Verfügung, die dazu dienen, einen Bedarf - etwa nach Rechercheleistungen - mit entsprechenden Angeboten zusammenzubringen. Insbesondere Studenten nutzen diese Möglichkeit, sich etwas hinzu zu verdienen, aber auch Freiberufler bessern auf diese Weise ihr Einkommen auf.

Bei der Frage danach, ob es für eine bestimmte Aufgabe sinnvoll ist, diese mittels Crowdsourcing zu lösen, ist ein wichtiger Aspekt die Vertraulichkeit. Wer zum Beispiel den Prototypen einer Software testen möchte, die dem eigenen Unternehmen einen entscheidenden Wettbewerbsvorteil verschaffen soll, sollte sich gut überlegen, ob Crowdsourcing dafür das richtige Mittel ist. Da die Crowd in der Regel relativ anonym ist, ist es kaum möglich, sicher auszuschließen, dass die Konkurrenz mitliest.

Viele Grüße,



Peter Pagel, Chefredakteur 